\title{
Midterm outcomes after aortic valve neocuspidization with glutaraldehyde-treated autologous pericardium
}

\author{
Shigeyuki Ozaki, MD, PhD, Isamu Kawase, MD, PhD, Hiromasa Yamashita, MD, Shin Uchida, MD, \\ Mikio Takatoh, MD, and Nagaki Kiyohara, MD
}

\section{ABSTRACT}

Objective: We had previously reported the short-term results of the aortic valve neocuspidization (AVNeo) procedure. We have now evaluated the midterm results with the longest follow-up of 118 months.

Methods: From April 2007 through December 2015, 850 patients were treated with AVNeo using autologous pericardium. Medical records of these patients were retrospectively reviewed. The procedure was on the basis of independent tricuspid replacement using autologous pericardium. The distances between the commissures were measured with an original sizing device, the pericardial cusp was trimmed using an original template, and then sutured to the annulus.

Results: There were 534 patients with aortic stenosis, 254 with aortic regurgitation, 61 with aortic stenoregurgitation, 19 with infective endocarditis, and 5 with a previous aortic valve procedure. Besides 596 patients with tricuspid aortic valve, 224 patients had bicuspid valve, 28 had unicuspid valve, and 2 had quadricuspid valve. There were 444 male and 406 female patients. The median age was 71 (range, 13-90) years old. Preoperative echocardiography revealed a peak pressure gradient average of $68.9 \pm 36.3 \mathrm{~mm} \mathrm{Hg}$ with aortic stenosis. Surgical annular diameter was $20.9 \pm 3.3 \mathrm{~mm}$. There was no conversion to a prosthetic valve replacement. There were 16 in-hospital mortalities. Postoperative echocardiography revealed a peak pressure gradient average of $19.5 \pm 10.3 \mathrm{~mm} \mathrm{Hg} 1$ week after surgery and $15.2 \pm 6.3 \mathrm{~mm} \mathrm{Hg} 8$ years after surgery. Fifteen patients needed reoperation (13 infective endocarditis, 1 break of thread, and 1 tear of cusp case). The mean follow-up period was $53.7 \pm 28.2$ months. Actuarial freedom from death, cumulative incidence of reoperation, and that of recurrent moderate aortic regurgitation or greater was $85.9 \%, 4.2 \%$, and $7.3 \%$, respectively, with the longest follow-up of 118 months.

Conclusions: The midterm outcomes of AVNeo using autologous pericardium were satisfactory in 850 patients with various aortic valve diseases. However, further randomized, multicenter prospective studies are needed to confirm the results of the current study. (J Thorac Cardiovasc Surg 2018;155:2379-87)

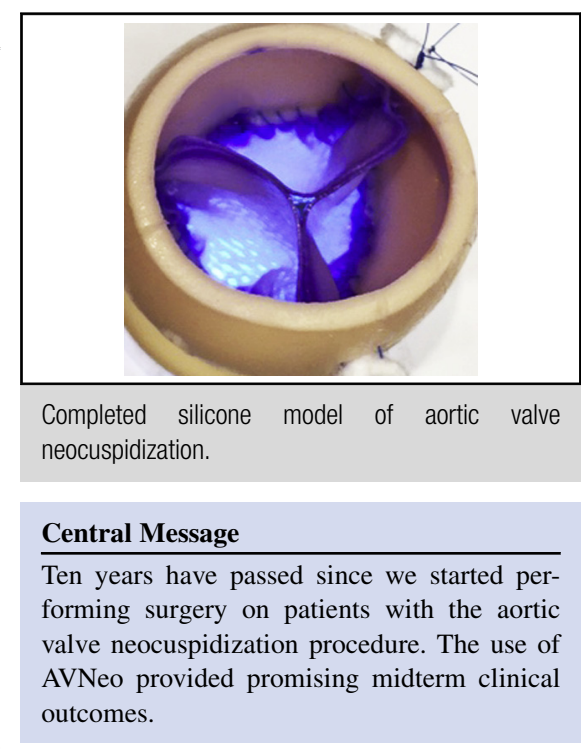

\section{Perspective}

Herein we report the feasibility of our original aortic valve neocuspidization using glutaraldehyde-treated autologous pericardium. Patients did not need anticoagulation postoperatively unless otherwise indicated. The midterm results with the longest followup of 118 months were satisfactory without serious complications. The echocardiographic follow-up proved maintained hemodynamics over time.

See Editorial Commentary page 2388.

See Editorial page 2377.

\footnotetext{
From the Department of Cardiovascular Surgery, Toho University Ohashi Medical Center, Tokyo, Japan.

Read at the 97th Annual Meeting of The American Association for Thoracic Surgery, Boston, Massachusetts, April 29-May 3, 2017.

Received for publication June 16, 2017; revisions received Dec 6, 2017; accepted for publication Jan 11, 2018; available ahead of print March 19, 2018.

Address for reprints: Shigeyuki Ozaki, MD, PhD, Department of Cardiovascular Surgery, Toho University Ohashi Medical Center, 2-17-6 Ohashi, Meguro-ku, Tokyo 152-8515, Japan (E-mail: ozakis@oha.toho-u.ac.jp). $0022-5223 / \$ 36.00$

Copyright (c) 2018 by The American Association for Thoracic Surgery https://doi.org/10.1016/j.jtcvs.2018.01.087
}

As the aging of the population becomes significant, we see more patients with coronary and peripheral artery diseases, aortic diseases, and atrioventricular valve diseases. For

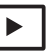

Scanning this QR code will take you to a supplemental video. To view the AATS Annual Meeting Webcast, see the URL next to the webcast thumbnail.

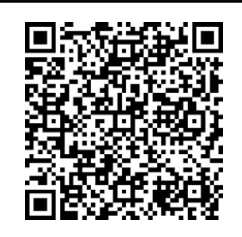




\section{Abbreviations and Acronyms \\ $\mathrm{AR}=$ aortic regurgitation \\ $\mathrm{AS}=$ aortic stenosis \\ AVR = aortic valve replacement \\ $\mathrm{AVNeo}=$ aortic valve neocuspidization \\ IE = infective endocarditis \\ PVE = prosthetic valve endocarditis}

aortic valve disease, bioprosthetic valves have been increasingly used for valve replacement, however, there are inherent problems in bioprosthesis such as cusp stiffening and calcification, and the risk of reoperation. ${ }^{1,2}$ Recently, transcatheter aortic valve replacement (AVR) has been used as a promising option for the patients with high-risk for open-heart surgery. ${ }^{3-5}$

Aortic valve neocuspidization (AVNeo) is the procedure we previously reported, which can be applied to a wide spectrum of aortic valve diseases, including aortic stenosis (AS), aortic regurgitation (AR), infective endocarditis (IE), prosthetic valve endocarditis (PVE), and annuloaortic ectasia. ${ }^{6,7}$ With this method, surgeons can preserve the natural aortic root expansion in systole with maximal effective orifice area, ${ }^{8,9}$ but they still replace the aortic valve as one whole structure. The difference in our original aortic valve reconstruction from others is the independent replacement of 3 cusps by 3 native-size autologous pericardial cusps. The sizes of each aortic cusp are defined by the distance between the commissures. ${ }^{6}$ We believe independent replacement of cusps is more effective in preserving the natural motion of the aortic valve annulus and the coordination of the left ventricle, aortic annulus, sinus of Valsalva, and aorta compared with conventional mechanical or bioprosthetic valves. The aim of this study was to report the clinical results of the patients with AVNeo with the longest follow-up of 118 months.

\section{METHODS}

Our AVNeo method and the clinical study of this procedure were approved by the institutional review board of Toho University Ohashi Medical Center. All patients underwent this procedure after written informed consent was obtained.

AVNeo was performed on 850 patients from April 2007 through December 2015. We retrospectively reviewed all 850 patients and evaluated midterm clinical results.

The indication of AVNeo was first by echocardiography for the patients with a small aortic annulus with a diameter $21.5 \mathrm{~mm}$, and second for patients who rejected mechanical valve implantation. Among them, we performed this reconstruction for patients who were willing to have this procedure.

As shown in Table 1, there were 444 male and 406 female patients. Median age was 71 (range, 13-90) years. Preoperative echocardiography showed an average peak pressure gradient through the aortic valve of $69.0 \pm 36.1 \mathrm{~mm} \mathrm{Hg}$ for patients with AS and a surgical annular diameter of $20.9 \pm 3.3 \mathrm{~mm}$ for all patients. Five hundred thirty-four patients had AS, 254 patients had AR, 61 patients had aortic stenoregurgitation. Among 19 patients with IE, 15 had AR, 3 cases had aortic stenoregurgitation, and 1 had lone IE with mobile vegetation refractory to antibiotics. The patient population consisted of 596 patients with tricuspid aortic valve, 224 patients with bicuspid aortic valve, 28 with unicuspid valve, and 2 with quadricuspid valve. Regarding the age distribution (Figure 1), most of the patients were older than 60 years, but there were younger patients as well.

\section{Surgical Technique (Video 1)}

The surgical technique for AVNeo has been reported previously. ${ }^{6}$ Preparation of autologous pericardium was started by cleaning fat and other redundant tissue on the outer surface of the pericardium with the Harmonic scalpel (Ethicon Endo-Surgery, Inc, Cincinnati, Ohio). The pericardium was excised at least $7 \times 8 \mathrm{~cm}$. The excised pericardium was then treated with $0.6 \%$ glutaraldehyde solution with a buffer for 10 minutes. The treated pericardium was rinsed 3 times using physiologic saline solution for 6 minutes.

Human pericardium is usually thin near the cranial end and thick caudally near the diaphragm. We use the thinner part of pericardium for small cusps to make the movement smoother, and the thicker part for large cusps to make them able to tolerate more stress. All procedures of AVNeo were performed during cardioplegic arrest on cardiopulmonary bypass.

First in the procedure, diseased cusps are excised meticulously. In case there is severe calcification along the aortic annulus, the Cavitron Ultrasonic Surgical Aspirator (SonoSurg, Olympus, Tokyo, Japan) is very helpful in removing calcium without damaging annular tissue. Then, the distance between each commissure is measured using a custom-made sizing apparatus. The intercommissural distance is measured using the custom-made sizing apparatus by giving the appropriate tension, similar to reproducing the annulus during diastole. Correct measurement is vital

TABLE 1. Baseline patient characteristics

\begin{tabular}{lc}
\hline \multicolumn{1}{c}{ Variable } & Value \\
\hline Mean age, y & $71(62-78)$ \\
Male sex & $444(52.2)$ \\
Hemodialysis patient & $101(11.8)$ \\
Surgery indication & \\
Aortic stenosis & $534(62.8)$ \\
Aortic regurgitation & $254(29.9)$ \\
ASR & $61(7.2)$ \\
Infective endocarditis & $19(2.2)$ \\
Reoperation after previous aortic valve surgery & $5(0.6)$ \\
Concomitant surgery & \\
AVNeo alone & $477(56.1)$ \\
Ascending aorta replacement & $113(13.3)$ \\
CABG & $84(9.9)$ \\
Mitral valve plasty & $66(7.8)$ \\
Mitral valve replacement & $32(3.8)$ \\
Tricuspid valve operation & $25(2.9)$ \\
Root replacement & $21(1.5)$ \\
Maze procedure & $85(10.0)$ \\
Myectomy & $22(2.6)$ \\
Other & $30(3.5)$ \\
Morphology of the aortic valve & \\
Tricuspid & $596(70.1)$ \\
Bicuspid & $224(26.4)$ \\
Unicuspid & $28(3.3)$ \\
Quadricuspid & $2(0.2)$ \\
\hline
\end{tabular}

Data are presented as $\mathrm{n}(\%)$ or mean (interquartile range). $A S R$, Aortic stenoregurgitation; $A V N e o$, aortic valve neocuspidization; $C A B G$, coronary artery bypass graft. 


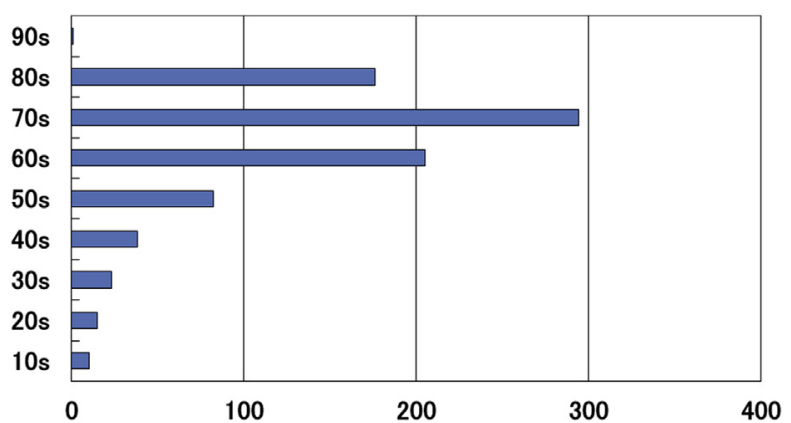

FIGURE 1. Age distribution of the patients: most of the patients were older than 60 years, but there was a significant number of younger patients.

to complete this procedure. The new cusp with the size corresponding to the measured value is trimmed with an original template from glutaraldehydetreated autologous pericardium. Last, the annular margin of the pericardial cusp is sutured with running 4-0 monofilament stitches to each annulus. The smooth (inner) surface of pericardium is placed on the left ventricular side. A running suture should be used to produce the natural, 3-dimensional bulge of the cusps effectively. The pericardial cusp is sewn thoroughly up to the top of commissure, and it is designed to have a deep coaptation that reaches up to the same horizontal plane as the commissure. The commissural coaptation is secured with additional 4-0 monofilament sutures together with a felt pledget at the commissure site. In cases with bicuspid valve, we used a 5-mm width felt strip (instead of felt pledget) to prevent future aortic dilatation as previously described. ${ }^{10}$ The coaptation of 3 new cusps is ensured with a visual check under negative pressure made by the left ventricular vent before closure of the aortotomy. For the sake of safety and reproducibility, AVNeo has evolved over time. From January 2008, the treated pericardium has been sutured with its smooth (serous) side facing the left ventricle to prevent platelet consumption. From January 2011, commissures have been sutured with a 5-mm width wing extension (as previously described). ${ }^{7}$ From November 2012, nontricuspid aortic valve cases have been treated with complete tricuspidization, in which we use existing raphe or create a new commissure to place 3 equal-sized cusps onto the annuls.

\section{Follow-up}

Low-dose aspirin was administered for 6 months after the procedure and discontinued unless necessary. All of the patients were monitored every 6 months at the outpatient clinic of our hospital or at the referral hospital. Echocardiographic evaluation has been done at 1 week, 1 month, 3 months, and every 6 months after surgery, and up to December 2015. Follow-up was performed either via hospital visit or telephone contact. If unable to be reached, the patient was classified as "lost follow-up." Regarding the clinical data such as death and reoperation, we updated them at the end of March 2017.

\section{Statistical Analysis}

Data are presented as mean \pm SD for continuous variables, and number $(\%)$ for categorical variables. Survival rate and cumulative incidents of reoperation or moderate or greater AR were calculated using the KaplanMeier method.

\section{RESULTS}

No surgery to convert to a prosthetic valve replacement was required. Surgery could be performed for aortic valve disease with IE and PVE in the same fashion. We performed AVNeo alone in $481(56.6 \%)$ cases. Hemiarch replacement

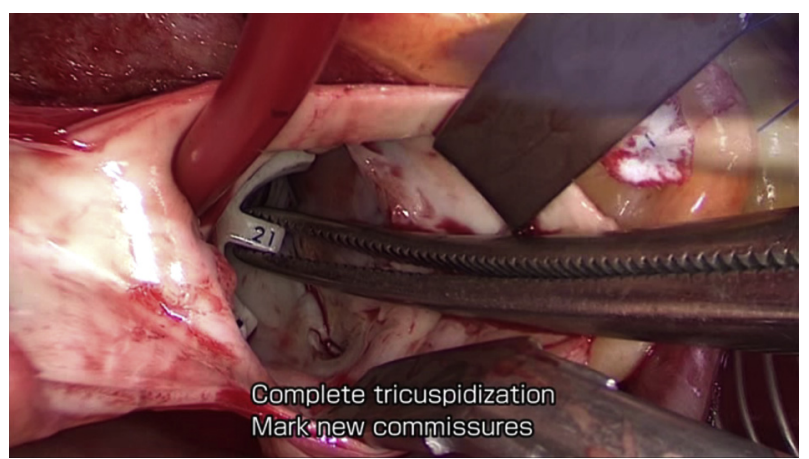

VIDEO 1. Procedures indicated according to time (minute:second) in the video. 00:12 to 00:28: Pre-transesophageal echocardiography. 00:13 to $00: 17$. Surgical annular diameter $=22 \mathrm{~mm}$. 00:31 to 00:35. Harvest pericardium. 00:35 to 00:39: Remove redundant fat tissue. 00:39 to 00:47: Treat pericardium with $0.6 \%$ glutaraldehyde for 5 minutes. 00:47 to 00:56: Rinse with normal saline for 6 minutes, 3 times (total of 18 minutes). 00:56 to 01:05. Aortotomy. 01:06 to 01:09: Expose using a retractor. 01:20 to 01:33: Sizing. Measure distance between the commissures or raphe $(17+19+27 / 3=21)$. 01:33 to 02:05: Complete tricuspidization. Mark new commissures. 02:06 to 02:49: Prepare the new pericardial cusps using the correct size template. 02:49 to 03:20: Mark out the new annulus. 03:20 to $03: 27$ : Suturing. $03: 48$ to $04: 22$ : Suture at $1-\mathrm{mm}$ intervals along the annulus starting from the midpoint to the commissure. 04:39 to 04:56: The big bite should be made at the second to last dot. 05:09 to 05:14: Straighten the needle. 05:14 to 05:19: The needle should penetrate $2 \mathrm{~mm}$ below the top of the commissure. 05:30 to 06:03: Suture commissure. 06:14 to 06:20: Before tying knots, make sure that the wing extensions are neatly against the aortic wall. 06:28 to 06:50: Reinforce new commissure using pericardial pledgets $(2 \times 3 \mathrm{~mm})$. 06:57 to 07:25: Adjust the length of the felt strips. 7:26 to 7:54: Pretransesophageal echocardiography, post-transesophageal echocardiography. Video available at: http://www.jtcvsonline.org/article/S0022-5223(18) 30371-4/fulltext.

was performed with AVNeo in 120 (14.1\%), and coronary artery bypass graft in $82(9.6 \%)$. For AR with annuloaortic ectasia, AVNeo could be combined effectively with an aortic root reimplantation technique such as the David procedure. Mean aortic cross-clamp time was $106.8 \pm 27.5$ minutes, and mean cardiopulmonary bypass time was $150.4 \pm$ 30.4 minutes for isolated aortic valve reconstructions.

Mean follow-up was $53.7 \pm 28.2$ months and the survival rate was $85.9 \%$ at 118 months (Figure 2, $A$ ), the cumulative incidence of reoperation was $4.2 \%$ (Figure $2, B$ ), and the cumulative incidence of recurrent $\mathrm{AR}$ or greater was $7.3 \%$. Table 2 shows a summary of the clinical outcomes after the AVNeo procedure. There were 101 deaths from any cause $(2.7 \%$ per patient-year). Among them, we observed 11 cardiovascular deaths $(0.3 \%$ per patientyear), 80 noncardiac deaths ( $2.1 \%$ per patient-year), and unknown 10 deaths $(0.3 \%$ per patient-year). Reoperations were performed in 15 patients $(0.4 \%$ per patient-year). IE 


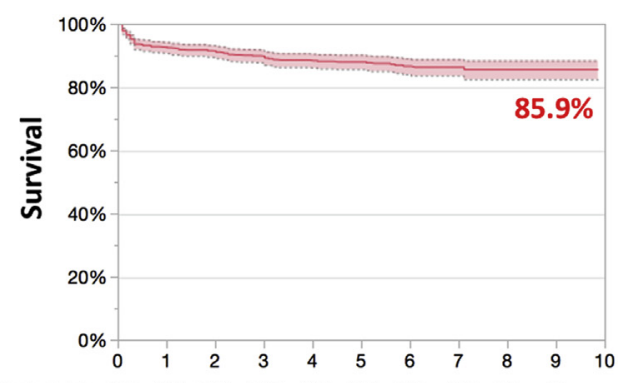

Patient at risk $850 \quad 788 \quad 714 \quad 604 \quad 482 \quad 377 \quad 262 \quad 132 \quad 56 \quad 15$ A

Years

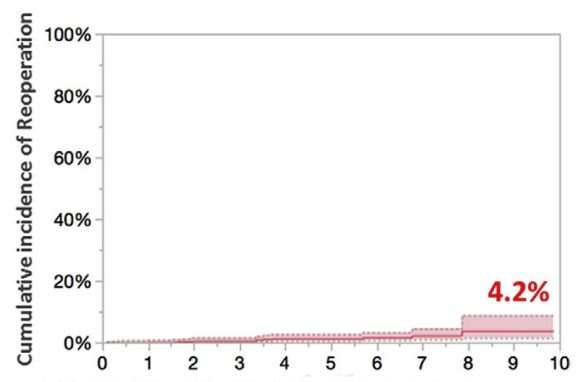

Patients at risk $850 \quad 784 \quad 707 \quad 600 \quad 475 \quad 371 \quad 256 \quad 128 \quad 54 \quad 14$

Years

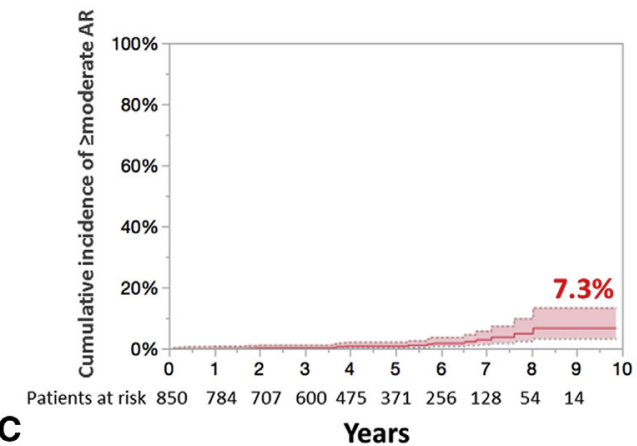

FIGURE 2. Kaplan-Meier curves of clinical outcomes. Shaded area indicates $95 \%$ confidence interval. A, Overall survival of the patients. B, Cumulative incidence of reoperation. $\mathrm{C}$, Cumulative incidence of recurrent moderate or greater aortic regurgitation.

was the reason for the reoperation in 13 cases, but the incidence was as low as $0.3 \%$ per patient per year. We observed 17 cases with echocardiography showing moderate or greater AR.

As shown in Table 3, the reason for reoperation was IE in 13 patients. These patients were treated with AVNeo using treated equine $(n=1)$ or bovine $(n=12)$ pericardium. One case had resuturing because of a tear of the thread on the noncoronary cusp 5 weeks after the surgery. Another case with a tear on the left coronary cusp itself 5 weeks after the surgery had 1-cusp reconstruction with bovine pericardium using AVNeo. To find the predisposing factors

TABLE 2. Clinical outcomes

\begin{tabular}{lc}
\hline \multicolumn{1}{c}{ Variable } & Value \\
\hline Death, n (\% per patient-year) & $101(2.7)$ \\
Cardiovascular death & $11(0.3)$ \\
Noncardiac death & $80(2.1)$ \\
Unknown death & $10(0.3)$ \\
Reoperation, n (\% per patient-year) & $15(0.4)$ \\
Infective endocarditis & $13(0.3)$ \\
Cusp tear & $1(0.03)$ \\
Others (break of thread) & $1(0.03)$ \\
Occurrence of more than moderate AR, & $17(0.5)$ \\
$\mathrm{n}(\%$ per patient-year) & \\
\hline$A R$, Aortic regurgitation.
\end{tabular}

related to the occurrence of IE, we added 2 post hoc analyses. When we divided cases into the tricuspid or the nontricuspid groups, the rate of IE was significantly higher in the nontricuspid group $(0.7 \%$ vs $3.5 \% ; P<.01)$. When we divided the cases before and after the introduction of the 5mm wing extension, the initial 289 cases (up to December 2010) and subsequent 561 cases (after January 2011) had similar incidence for IE ( $0.4 \%$ vs $0.3 \%$ per patient-year).

Preoperative average peak pressure gradient through the aortic valve with echocardiography was $68.9 \pm 36.3 \mathrm{~mm}$ $\mathrm{Hg}$. The pressure gradient decreased $19.5 \pm 10.3 \mathrm{~mm} \mathrm{Hg}$ at 1 week after surgery, $14.0 \pm 6.6 \mathrm{~mm} \mathrm{Hg}$ at 1 year after surgery, and $15.2 \pm 6.3 \mathrm{~mm} \mathrm{Hg}$ at 8 years after surgery (Figure 3). Figure 4 shows a representative case of a 14year-old male patient with a failed unicuspid aortic valve. Echocardiographic follow-up 7 years after the procedure showed no signs of calcification nor AR, and natural motion of the 3 cusps.

\section{DISCUSSION}

It has been 10 years since we started to perform surgery on patients using AVNeo. As with our previous report in 404 patients, the clinical outcomes after a longer followup with more patients were still favorable, however, we experienced a total of 15 cases with reoperation: 13 cases with IE, 1 with a tear of the cusp, and 1 with a break of the thread. From the findings of echocardiography, we 
TABLE 3. Details of the cases with reoperation after AVNeo

\begin{tabular}{|c|c|c|c|c|}
\hline Age, y/sex & Surgical indication & $\begin{array}{c}\text { Timing of } \\
\text { reoperation, mo }\end{array}$ & Reason for reoperation & Time of initial AVNeo \\
\hline $72 / \mathrm{M}$ & AS & 42 & IE: vegetation and calcification on RCC, large hole on NCC & February 2008 \\
\hline 70/M & AS & 40 & IE: vegetation and hole on RCC & April 2009 \\
\hline 57/M & AS (BAV) & 11 & IE: vegetation and hole on NCC & January 2010 \\
\hline $53 / \mathrm{F}$ & AS (BAV) & 20 & IE: vegetation on LCC & May 2010 \\
\hline $80 / \mathrm{F}$ & AS (BAV) & 18 & IE: vegetation and hole on LCC, vegetation on NCC & February 2011 \\
\hline 37/M & AS (BAV) & 40 & IE: vegetation and hole on NCC & September 2011 \\
\hline 64/M & AS (BAV) & 23 & IE: vegetation and hole on NCC, vegetation on RCC & March 2012 \\
\hline $59 / \mathrm{F}$ & AS (BAV) & 23 & IE: vegetation and hole on LCC & July 2012 \\
\hline 63/M & AS (BAV) & 94 & IE: vegetation on $\mathrm{LCC}$ and $\mathrm{RCC}$ & November 2007 \\
\hline 73/M & AS & 81 & IE: vegetation and hole on NCC & May 2008 \\
\hline 68/M & AR & 68 & IE: vegetation and hole on all cusp & March 2010 \\
\hline $54 / \mathrm{M}$ & AS (UAV) & 55 & IE: vegetation on $\mathrm{RCC}$ and $\mathrm{NCC}$ & October 2010 \\
\hline $77 / \mathrm{F}$ & $\mathrm{AR}$ & 1 & Tear of the thread of NCC & November 2014 \\
\hline $54 / \mathrm{M}$ & AS (BAV) & 4 & IE: root abscess on RCC, vegetation on LCC and RCC & November 2014 \\
\hline 33/M & $\mathrm{AR}$ & 1 & Tear ( $5 \mathrm{~mm}$ in size) on LCC close to RCC commissure & December 2015 \\
\hline
\end{tabular}

$A V N e o$, Aortic valve neocuspidization; $M$, male; $A S$, aortic stenosis; $I E$, infective endocarditis; $R C C$, right coronary cusp; $N C C$, noncoronary cusp; $B A V$, bicuspid aortic valve; $F$, female; $L C C$, left coronary cusp; $A R$, aortic regurgitation; $U A V$, unicuspid aortic valve.

observed a stable pressure gradient and low rate of recurrent AR after AVNeo throughout the follow-up period.

In this report, we are able to present cases with reoperation after AVNeo. As we mentioned in the results, the incidence rate of IE necessitating reoperation was $0.3 \%$ per patient-year. On the other hand, the rates of postoperative IE after AVR with bioprosthetic valves ranged from 0.8 to $1.0 \%$ per patient-year. ${ }^{11-13}$ Regarding the structural problems related to the valve itself, we only had one reoperation case of 850 cases. Although our cohort lacks

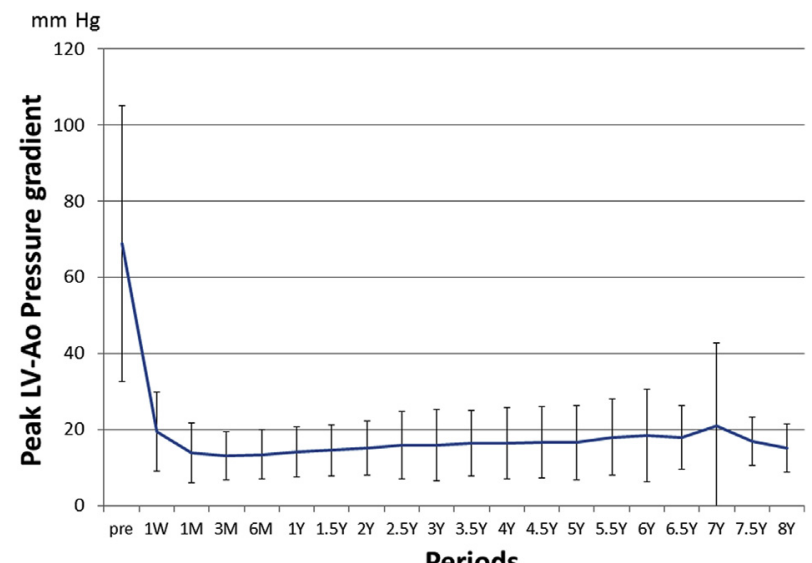

FIGURE 3. Pre- and postoperative average peak pressure gradient through the aortic valve with echocardiography. There was no increase of the pressure gradient up to 8 years in the overall cohort. $L V$-Ao, Left ventricle-ascending aortic. a long-term (ie, 10-15 years) follow-up, we observe good results for the moment.

We have updated the procedure 3 times to obtain better results: (1) at the beginning, we used the rough surface of the treated pericardium faced to the left ventricle and we observed significant decrease in the platelet counts in certain cases. After January 2008, we changed the side of the treated pericardium and found no cases with platelet count decrease postoperatively; (2) a 5-mm width wing extension was introduced in January 2011, resulting in a better fixation of the cusp around the commissures; and (3) tricuspidization of the nontricuspid case using preexisting commissures had been performed for 5.5 years, however, there were several cases in which sizes of 3 cusps were very different, in which postoperative echocardiography showed uneven movements of the cusps. Therefore, from November 2012, we started complete tricuspidization with 3 equal-sized cusps. Since then, we have been getting better movements of the cusps according to postoperative echocardiography. To evaluate these 3 changes, we performed several analyses comparing the clinical results before and after the introduction of these modifications, but found no significant differences. Statistically speaking, we need more events and longer follow-up to get the meaning of these changes.

Bioprosthetic valves have been reported to have structural valve deterioration later in the follow-up, especially in the younger population. ${ }^{14,15}$ Although there are a number of younger patients in our cohort, we have yet to see patients with structural problems of the reconstructed 


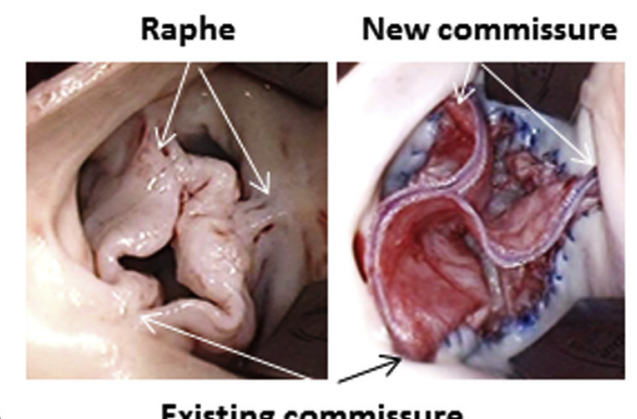

A

$$
\text { Existing commissure }
$$
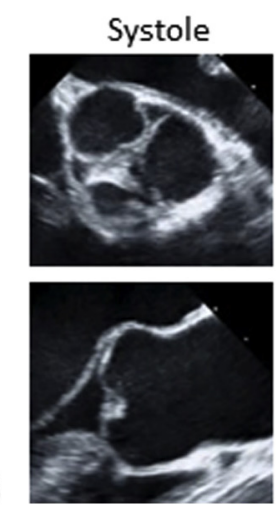

Diastole
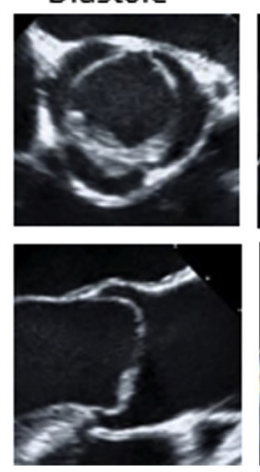
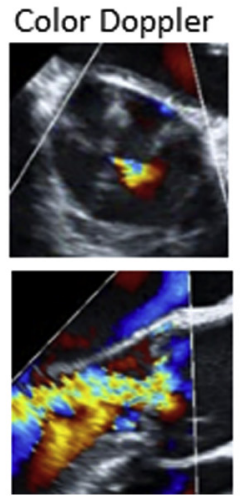

C
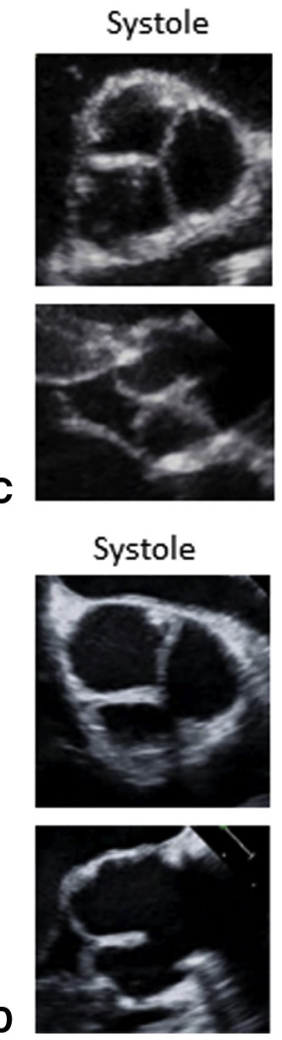

Diastole
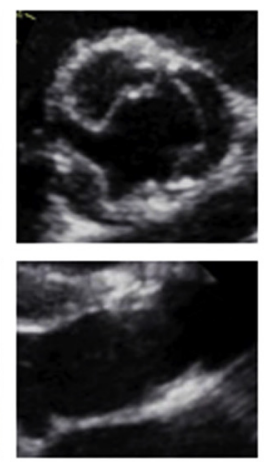

Diastole
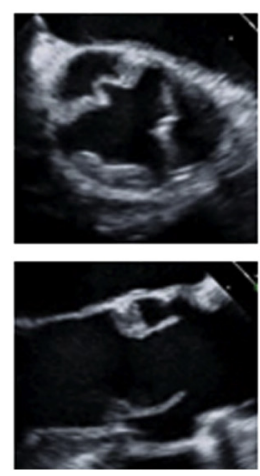

Color Doppler
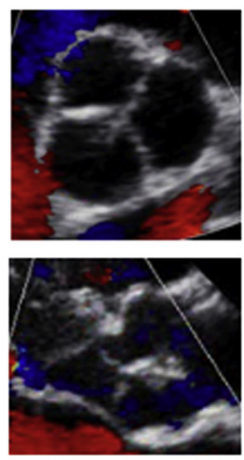

Color Doppler
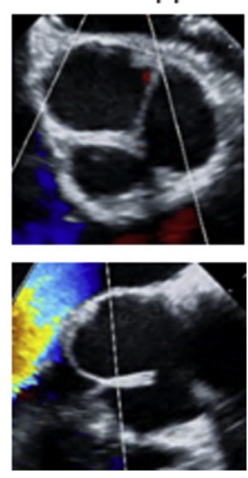

FIGURE 4. A representative case. We performed our original aortic valve reconstruction on a 14-year-old boy with a failed unicuspid aortic valve. A, Left image shows the pretreatment unicuspid valve and right image shows the reconstructed aortic valve after AVNeo. B, Preoperative transesophageal echocardiography showed significant aortic regurgitation with restricted opening of the aortic valve. C, One-month follow-up transesophageal echocardiography showed no sign of aortic regurgitation and a fully opened aortic valve. D, Transesophageal echocardiography 7 years after the surgery still showed the good results and no sign of degeneration of the aortic valve.

valve. We showed a case of a 14-year-old male patient in Figure 4 suggesting the possibility of AVNeo's durability even in younger patients.

Theoretical backgrounds of the durability of our reconstructed valve are described in this paragraph. First, we calculate cusp free-edge length from the intercommissural length and the contact point of the new pericardial cusps are raised up to the same plane of the commissures. With these concepts, we are able to have a larger coaptation zone and decrease the mechanical stress onto the commissures and annulus. Second, independent replacement of cusps is effective in preserving the natural motion of the aortic valve annulus and the coordination of the left ventricle, aortic annulus, sinus of Valsalva, and aorta. With our technique of total resection of all 3 diseased cusps, AVNeo can be applied to any type of aortic valve disease. In fact, we can operate on patients with AS, AR, IE, and PVE, and those with nontricuspid aortic valve, basically in the same fashion. Third, the treated pericardium has been used to reconstruct the aortic valve. Glutaraldehyde treatment provides more resistance against retraction and degeneration, and maintains intrinsic tissue pliability of the pericardium. The ultimate tensile strength of glutaraldehyde-treated human pericardium was 4 times greater than noncalcified leaflets, indicating its suitability for application to aortic valve reconstruction. ${ }^{16}$

There are several clinical reports from other institutions. Rosseykin et al reported that significantly lower values of the aortic valve pressure gradient and larger effective orifice area of the aortic valve were observed on postoperative echocardiography in patients with AVNeo compared with those with conventional bioprosthetic valves. ${ }^{17}$ According to the report by Yamamoto et al, aortic annular changes in patients with AVNeo were similar to those with normal aortic valves. This was evidenced by electrocardiography-gated multidetector computed tomography, previously reported as the most reliable method, to evaluate annulus motion during the cardiac cycle. They also reported less pressure gradient in patients with AVNeo than in those with AVR. ${ }^{18}$ Yoshikawa et al have reported the usefulness of measurements of the aortic annulus diameter using speckle-tracking transesophageal echocardiography. Figure 5 shows 3 measurements during 

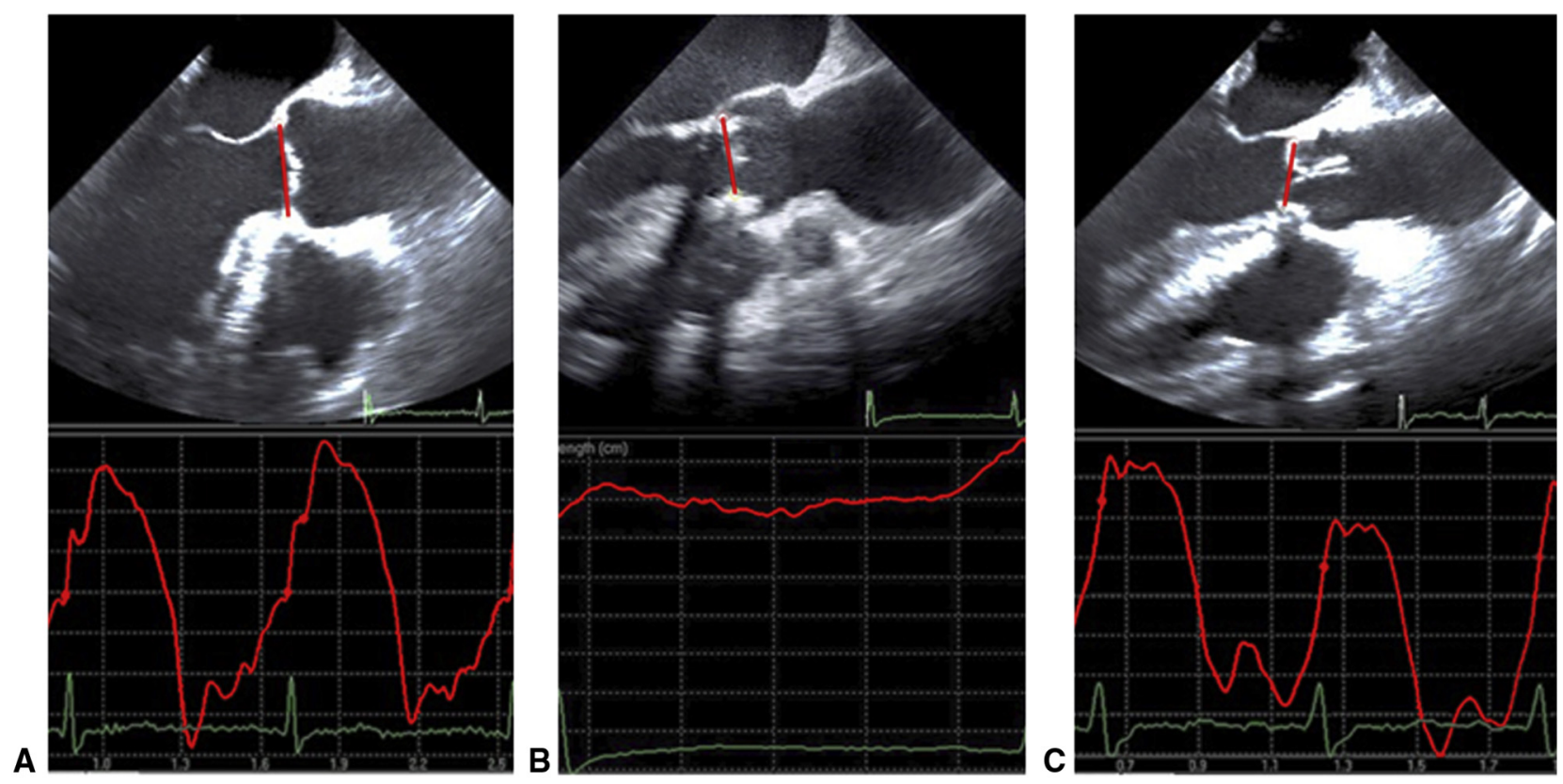

FIGURE 5. Deformation dynamics and mechanical properties of the aortic annulus using transesophageal echocardiography with the speckle-tracking method. A, A case with a normal aortic valve had a dynamic change in the diameter of the aortic root. B, A case with AVR had a fixed diameter due to the stent implanted. C, A case with AVNeo had a maintained change in the aortic root diameter similar to that in the normal case.

the cardiac cycle. Compared with the measurement in an AVR case, systolic expansion of the aortic annulus is well maintained in a case with AVNeo. ${ }^{19}$ This evidence shows that aortic annulus after AVNeo maintains natural movement and reconstructed aortic valve has significantly less pressure gradients compared with the cases with conventional AVR.

\section{Study Limitation}

The present study is a retrospective single-center study, therefore, the results might be different if performed in other institutions. Although the study shows the midterm results with the longest follow-up of 118 months, long-term results have to be studied with a longer follow-up of 10 to 15 years. There are possibilities that biocompatible materials other than autologous pericardium might also provide a good result, however, we will need clinical trials to prove it. Although our cardiopulmonary bypass time and aortic cross-clamp time were longer than those in standard AVR, we achieved clinical outcomes comparable with conventional therapies with good hemodynamics and a warfarinfree condition.

\section{CONCLUSIONS}

AVNeo provided favorable midterm results in 850 patients with various aortic valve diseases. Multicenter longterm studies are warranted in the future to prove the validity of this procedure.

\section{Webcast}

You can watch a Webcast of this AATS meeting presentation by going to: https://aats.blob.core.windows.net/media/ 17AM/2017-05-03/RM302-304/05-03-17_Room302-304_ 0742_Ozaki.mp4.

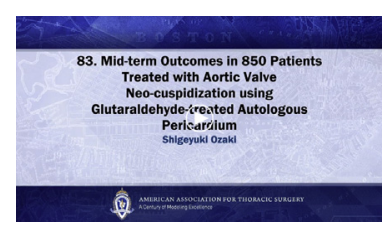

\section{Conflict of Interest Statement}

Dr Ozaki is a consultant for the Japanese Organization for Medical Device Development, Inc. All other authors have nothing to disclose with regard to commercial support.

\section{References}

1. Ozaki S. Pathophysiology of Calcification of Bioprosthetic Heart Valves: An Experimental Investigation. Leuven, Belgium: Leuven University Press; 2001.

2. Hammermeister K, Sethi GK, Henderson WG, Grover FL, Oprian C, Rahimtoola SH. Outcomes 15 years after valve replacement with a mechanical versus a bioprosthetic valve: final report of the Veterans Affairs randomized trial. J Am Coll Cardiol. 2000;36:1152-8.

3. Smith CR, Leon MB, Mack MJ, Miller DC, Moses JW, Svensson LG, et al. Transcatheter versus surgical aortic-valve replacement in high-risk patients. $N$ Engl J Med. 2011;364:2187-98.

4. Reinöhl J, Kaier K, Reinecke H, Schmoor C, Frankenstein L, Vach W, et al. Effect of availability of transcatheter aortic-valve replacement on clinical practice. N Engl J Med. 2015;373:2438-47. 
5. McCarthy FH, Spragan DD, Savino D, Dibble T, Hoedt AC, McDermott KM, et al. Outcomes, readmissions, and costs in transfemoral and alterative access transcatheter aortic valve replacement in the US Medicare population. J Thorac Cardiovasc Surg. 2017;154:1224-32.e1.

6. Ozaki S, Kawase I, Yamashita H, Uchida S, Nozawa Y, Matsuyama T, et al. Aortic valve reconstruction using self-developed aortic valve plasty system in aortic valve disease. Interact Cardiovasc Thorac Surg. 2011;12:550-3.

7. Ozaki S, Kawase I, Yamashita H, Uchida S, Nozawa Y, Takatoh M, et al. A total of 404 cases of aortic valve reconstruction with glutaraldehyde-treated autologous pericardium. J Thorac Cardiovasc Surg. 2014;147:301-6.

8. Cheng A, Dagum P, Miller DC. Aortic root dynamics and surgery: from craft to science. Philos Trans R Soc Lond B Biol Sci. 2007:362:1407-19.

9. Rodríguez F, Green GR, Dagum P, Nistal JF, Harrington KB, Daughters GT, et al. Left ventricular volume shifts and aortic root expansion during isovolumic contraction. J Heart Valve Dis. 2006;15:465-73.

10. Ozaki S, Kawase I, Yamashita H, Uchida S, Nozawa Y, Takatoh M, et al. Reconstruction of bicuspid aortic valve with autologous pericardium. Circ J. 2014;78: 1144-51.

11. Arvay A, Lengyel M. Incidence and risk factors of prosthetic valve endocarditis. Eur J Cardiothorac Surg. 1988;2:340-6.

12. Hartz RS, Fisher EB, Finkelmeier B, DeBoer A, Sanders JH, Moran JM, et al. An eight-year experience with porcine bioprosthetic cardiac valves. J Thorac Cardiovasc Surg. 1986;91:910-7.

13. Poirer NC, Pelletier LC, Pellerin M, Carrier M. 15-Year experience with the Carpentier-Edwards pericardial bioprosthesis. Ann Thorac Surg. 1998;66(6 Suppl):S57-61

14. Johnston DR, Soltesz EG, Vakil N, Rajeswaran J, Roselli EE, Sabik JF III, et al. Long-term durability of bioprosthetic aortic valves: implications from 12,569 implants. Ann Thorac Surg. 2015;99:1239-47.

15. Jamieson WR, Burr LH, Miyagishima RT, Germann E, MacNab JS, Stanford E, et al. Carpentier-Edwards supra-annular aortic porcine bioprosthesis: clinical performance over 20 years. J Thorac Cardiovasc Surg. 2005;130:994-1000.

16. Yamashita H, Ozaki S, Iwasaki K, Kawase I, Nozawa Y, Umezu M. Tensile strength of human pericardium treated with glutaraldehyde. Ann Thorac Cardiovasc Surg. 2012;18:434-7.

17. Rosseykin EV, Bazylev VV, Batrakov PA, Karnakhin VA, Rastorguev AA. Immediate results of aortic valve reconstruction by using autologous pericardium (Ozaki procedure). Patol krovoobrashch kardiokhir. 2016;20:44-8.

18. Yamamoto Y, Iino K, Shintani Y, Kato H, Kimura K, Watanabe G, et al. Comparison of aortic annulus dimension after aortic valve neocuspidization with valve replacement and normal valve. Semin Thorac Cardiovasc Surg. 2017;29:143-9.

19. Yoshikawa H, Suzuki M, Hashimoto G, Kusunose Y, Otsuka T, Hara H, et al. Assessment of cyclic changes in the diameter of the aortic annulus using speckle-tracking trans-esophageal echocardiography. Ultrasound Med Biol. 2013;39:2084-90.

Key Words: aortic valve disease, autologous pericardium, aortic valve surgery

\section{Discussion}

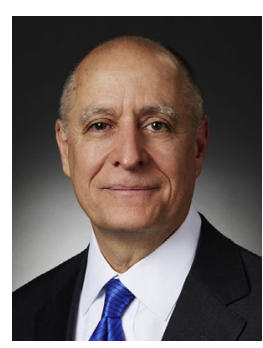

Dr J. Michael DiMaio (Dallas, Tex). Thank you, Dr Mohr, Dr Smith, Dr Barlow, and the Association for inviting me to discuss this paper. Professor Ozaki, I want to thank you as well for a very nice presentation, well done, and for sending me the manuscript well in advance.

First, I want to congratulate you for dedicating yourself to a new procedure, continuing to analyze it, and sharing the results in a public, peer-reviewed manner. I appreciate you taking the time to travel around the world to teach others how to perform this procedure, including our institution in Dallas. At the Heart Hospital at Baylor, Drs
Brinkman, Harrington, Ryan, and I thoroughly enjoyed your visit where you carefully showed us your technique. We, like other high-volume centers doing aortic surgery, strive to save or repair as much aorta and valve tissue as possible, of course depending on the disease state. Your technique is another possible option in these patients. In this audience, Dr Rankin is also another example of people who are trying to save tissue as much as possible.

I have a few questions. I will ask them one at a time and allow you to answer one before I ask the next question. First, operative procedure. Previously you reported in The Journal of Thoracic and Cardiovascular Surgery in 2014 the first 400 or so patients that you had achieved an ischemic time over the duration of 6 months of about 96 minutes for your procedure. In this article in this series you are now reporting a mean cross-clamp time of 107 minutes. What accounts for this additional time? One would expect with your templates and more experience they would shorten. Are you teaching the procedure more often, are observers adding increased time, are residents or fellows being involved more in these cases? What other changes have occurred taking this increased time to perform the procedure?

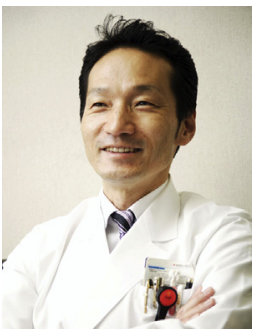

Dr Shigeyuki Ozaki (Tokyo, Japan). Thank you very much for your question. There are 2 reasons. First is that, as you mentioned, my training opportunity is increasing, and also my younger staff is studying my procedure, and the second is, I mentioned in my presentation, we updated the procedure. Since November 2012 we started to do the complete tricuspidization. We are thinking a new commissure on the sinus of Valsalva is weaker than the existing commissure. So we always reinforce the commissure using a small pericardial strip on the new commissure. The aortic wall and the wing extension is sandwiched with small pericardial strips inside and outside. So it takes more time compared with previously. That's the reason. Thank you.

Dr Dimaio. So the added steps in the technique have increased the time.

Next, valve thrombosis. This is a pertinent topic given the findings of several investigations of transcatheter heart valves in the literature recently. In fact, at our institution we currently have a protocol utilizing 4D CT scanning after development of increased gradients or decreased EF findings at the time of postoperative echo evaluation. This is the newest modality that might give an early warning sign that thrombosis is occurring and allow more aggressive anticoagulation.

You mentioned one advantage of your procedure is the ability to keep patients off of warfarin. Do you have any concerns about the valve thrombosis on your neoaortic 
valves? Have you seen any signal of clinical or subclinical valve thrombosis via echo studies or the more recent scanning such as 4D CT? Has the information coming out about TAVR valve thrombosis made you consider anticoagulating these patients at least for a few months after the procedure?

Dr Ozaki. Thank you very much for your suggestion. Unfortunately, we don't have 4D CT scanning in my institution, but we are always checking the motion of the cusp after operation using transesophageal echocardiography. We saw that some patients have a restrictive motion of the cusp. Our cardiologist is deciding the reason why, but we haven't an answer yet. But in the patient with restrictive motion of the cusp, there is no clinical sign of thrombosis. Also, we had 15 redo cases. During the redo surgery we always sew the cusp, but we haven't seen the thrombosis on the cusp. So probably that is why we are giving only aspirin for 6 months. Thank you.

Dr Dimaio. Next, imaging for leaflet sizing. Could it be possible to use preoperative imaging to make the necessary measurements and then go into the procedure with the neoleaflets already sized and cut and therefore ready to be sewn to decrease the operative time?
Dr Ozaki. I completely agree with you. If we can measure the distance between the commissures exactly before operation, I think we can decrease the cross-clamping time.

Dr Dimaio. My last question. In terms of materials, you used autologous pericardium. When you came to the United States and helped train us, we used another material, CardioCel, in that material. Have you considered other materials in addition to autologous pericardium in your series?

Dr Ozaki. Yes. In Japan we don't have CardioCel or PhotoFix, this sort of material, but if we had it, I'd like to use it. When we go to several countries, we have a chance to use these materials, but I haven't the long-term results yet. But among the 850 patients, we used 28 with treated pericardium; 23 is bovine, 5 is occurrence post pericardium. Longest follow-up is 9 years, but the result is quite good; freedom from reoperation is $100 \%$. If we can't use the autograft pericardium, we will use treated bovine pericardium in Japan.

Dr Dimaio. Professor Ozaki, thank you again for your dedication to the procedure and sharing it with many of us and presenting it at the AATS. 